Eine ausdrückliche Zustimmung des Antragstellers, die Bibliothek unter Kulturgutschutz zu stellen, liegt hier nicht vor. Es ist ein Irrglaube, dass alle Leihgaben an öffentliche Einrichtungen automatisch nationales Kulturgut seien. Dazu muss entweder bei Leihgabe oder nachträglich die ausdrückliche Zustimmung des Leihgebers eingeholt werden - ein Umstand, den beispielsweise die öffentlichen Museen bisher nicht berücksichtigen. Die Beweislast für eine solche Zustimmung liegt bei demjenigen, der sich darauf beruft.

Selbst wenn der Leihgeber seine Zustimmung erteilt hat, kann er sie nach $\S 6$ Abs. 2 S. 2 KGSG jederzeit widerrufen und spätestens endet sie nach § 6 Abs. 2 S. 4 KGSG mit der Kündigung des Leihvertrages. In dem Abzug der Sammlung aus der Institutsbibliothek durch den Antragsteller liegt wohl eine Kündigung des Leihvertrages, so dass spätestens ab diesem Zeitpunkt die Bibliothek, sollte sie noch im Privatbesitz stehen, kein nationales Kulturgut mehr wäre. Eine Ausfuhrgenehmigung hätte dann erteilt werden müssen.

Hat der Antragsteller dagegen die Schenkung vollzogen und wurde die Bibliothek dadurch Eigentum der Universität, so ist sie nationales Kulturgut nach § 6 Abs. 1 Nr. 2 KGSG. Eine Ausfuhr wäre dann ohne Genehmigung nicht erlaubt. Dies schon allein deshalb, weil der Antragsteller dann nicht mehr verfügungsberechtigt gewesen wäre.

Allerdings sprechen auch hier die Erwägungen des Kulturgutschutzes nicht gegen eine Ausfuhr. Nach § 23 Abs. 2 KGSG hätte eine Ausfuhrgenehmigung für nationales Kulturgut nur dann versagt werden dürfen, wenn bei Abwägung der Umstände des Einzelfalls wesentliche Belange des deutschen Kulturgutbesitzes überwogen hätten. Die oben im Rahmen des § 7 Abs. 1 KGSG angestellten Erwägungen zur identitätsstiftenden Wirkung der Bibliothek wären auch hier anzustellen. Selbst wenn die Bibliothek also als Bestand einer öffentlichen Einrichtung nationales Kulturgut gewesen wäre, hätte eine Ausfuhrgenehmigung erteilt werden müssen, die dem Grundgedanken des Kulturgüterschutzes nicht entgegengestanden hätte, denn identitätsstiftend für die Kultur Deutschlands ist die Bibliothek nicht.

Das zeigt, dass die Belange des Kulturgutschutzes einen Verbleib der Bibliothek in Deutschland nicht verlangen. Bei dem gesamten Streit geht es hier also allein um die Frage, wer Eigentümer der Bibliothek ist - die Universität oder der Antragsteller. Muss dazu der Kulturgutschutz bemüht werden?

\title{
Rechtsprechung
}

\section{Sichergestellte Bücher der Arabisch-Islamischen Wissenschaften dürfen vorläufig nicht ausgeführt werden}

VG Frankfurt a.M. Beschl. v. 01.02.2018, Az.: 5 L 5640/17.F

\section{Tenor:}

Der Antrag wird abgelehnt.

Die Kosten des Verfahrens einschließlich der außergerichtlichen Kosten der Beigeladenen hat der Antragsteller zu tragen.

Der Streitwert wird auf 25000 Euro festgesetzt.

\section{Gründe}

\section{I.}

[1] Die Beteiligten streiten über die Anordnung der aufschiebenden Wirkung der Klage des Antragstellers gegen die Sicherstellung von Bibliotheks- und Sammlungsbeständen nach dem Kulturgutschutzgesetz (KGSG) vom 31. Juli 2016 (BGBI. I S. 1914), das durch Art. 6 Abs. 13 des Gesetzes vom 13. April 2017 (BGBI. I S. 872) - FNA 224-26 - geändert worden ist.
[2] Der inzwischen 93-jährige Antragsteller war Mitbegründer und langjähriger geschäftsführender Direktor der im Jahre 1981 gegründeten Beigeladenen, einer Stiftung bürgerlichen Rechts, die Vermögensträger und Verwalter des Instituts für Geschichte der Arabisch-Islamischen Wissenschaften an der Johann Wolfgang Goethe-Universität in Frankfurt a.M. ist und den Zweck verfolgt, der Erforschung und Lehre der Geschichte der Wissenschaften im arabisch-islamischen Kulturraum zu dienen. Die Vertretung des Antragsgegners ist die nach $\S 3$ Abs. 1 S. 2 KGSG in Verbindung mit $\S 1$ der Verordnung zur Benennung der zuständigen Behörde nach dem Kulturgutschutzgesetz vom 24. Oktober 2016 (GVBI. S. 186) - FFN 75-3 - zuständige Behörde.

[3] Die Bibliothek des Instituts enthält dessen Internetauftritt https://www.uni-frankfurt.de/58601604/IGAIW zufolge über 25 000 Bände, etwa 300 arabische Handschriften und Mikrofilme von ca. 7000 Handschriften mit wichtigen Texten und Studien aus den arabischen Geistes- und Naturwissenschaften, eine vollständige Sammlung der Kataloge arabischer Handschriften in aller Welt und Exemplare der meisten Fachzeitschriften auf den Gebieten Orientalistik und Islamkunde sowie allgemeine 
Wissenschaftsgeschichte in europäischen Sprachen und auf Arabisch mit einem Sammelschwerpunkt auf der arabischislamischen Geographie. Darüber, wer welche Rechte an diesem Bestand hat, herrscht zwischen den Beteiligten Streit. Der Antragsteller ist der Ansicht, ca. 20000 Bände gehörten ihm und seiner Frau; eine zeitweise erwogene Schenkung sei nicht vollzogen worden. Da sich für den Antragsteller aus seiner Sicht bessere Forschungsmöglichkeiten in der Türkei boten, beabsichtigte er, den Großteil des ihm gehörigen Bestands in die KStiftung in Istanbul einzubringen. Eine erste Ladung ging am 4. Mai 2017 über den Flughafen Frankfurt a.M. nach Istanbul, eine zweite wurde am 6. Mai 2017 durch das Zollfahndungsamt Frankfurt a.M. sichergestellt.

[4] Mit der hier streitgegenständlichen Verfügung des Hessischen Ministeriums für Wissenschaft und Kunst vom 12. Mai 2017 (BI. 59, 60 = BI. 73, 74 d.A.) wandte sich dieses an das Polizeipräsidium Frankfurt a.M., verfügte „bis auf weiteres" die Sicherstellung der Bibliothek, der Bibliotheks- und Sammlungsgegenstände, die in den Räumen des Instituts für Geschichte der Arabisch-Islamischen Wissenschaften an der Johann Wolfgang Goethe-Universität, Westendstraße 89, Frankfurt a.M., lagern und ordnete an, dass diese für die Zeit der Sicherstellung in den Räumen des Instituts verblieben. Noch am Abend des 12. Mai 2017 wurde diese Verfügung von Polizeivollzugsbeamten und Beamten des Zollfahndungsamts vor Ort vollzogen. Die Präsidentin der Johann Wolfgang Goethe-Universität beantragte mit Schreiben ebenfalls vom 12. Mai 2017 an das Hessische Ministerium für Wissenschaft und Kunst die Einleitung des Verfahrens auf Eintragung in ein Verzeichnis national wertvollen Kulturguts nach § 14 Abs. 1 KGSG. Mit Schreiben vom 15. Mai 2017 meldeten sich die Bevollmächtigten des Antragstellers beim Antragsgegner und machten geltend, die Verfügung sei nicht rechtmäßig und deshalb umgehend aufzuheben. Eine Begründung seiner Verfügung gab das Hessische Ministerium für Wissenschaft und Kunst mit Schreiben vom 16. Mai 2017 (BI. $56-58=$ BI. $75-77$ d.A.) nachträglich.

[5] Mit Schriftsatz seiner Bevollmächtigten vom 12. Juni 2017 hat der Antragsteller am 12. Juni 2017 vor dem Verwaltungsgericht Frankfurt a.M. Klage erhoben, die unter der Geschäftsnummer 5 K 5641/17.F geführt wird und über die bislang nicht entschieden ist, sowie beantragt, deren aufschiebende Wirkung anzuordnen.

[6] Der Antragsgegner verteidigt die angegriffene Verfügung und wendet im Wesentlichen ein, es handele sich um ein Kulturgut, für das in seiner Sachgesamtheit ein Ausfuhrverbot in die Türkei als Drittstaat nach der Verordnung (EG) Nr. 116/2009 bestehe. Zudem seien die Eigentumsverhältnisse am Bibliotheksgut unklar.

[7] Die Beigeladene verteidigt - nach einstweiliger Untersagung der Tätigkeit des Antragstellers als geschäftsführender Direktor durch Bescheid des Regierungspräsidiums Darmstadt vom 20. Juni 2017 (Bl. 100 - 109 d.A.) und Bestellung eine Not- vorstands aufgrund Antrags des Regierungspräsidiums Darmstadt vom 12. Juli 2017 (BI. 117, 118, 120, 119 d.A.) durch Beschluss des Amtsgerichts Frankfurt a.M. vom 26. September 2017 - 75 AR 7/17 - (BI. 140 - 142 = BI. 159, 160 d.A.) - ebenfalls die angegriffene Verfügung.

[8] Die Staatsanwaltschaft Frankfurt a.M. hat durch Verfügung vom 8. September 2017 - 8940 Js 221296/17 - (BI. 125, 128 - 135 d.A.) ein Ermittlungsverfahren gegen den Antragsteller wegen Verdachts der Unterschlagung nach § 246 StGB und Verstoßes gegen § 83 KGSG nach § 170 Abs. 2 StGB eingestellt.

\section{II.}

[9] Der zulässigerweise gestellte Antrag, die von Gesetzes wegen nach § 80 Abs. 2 S. 1 Nr. 3 VwGO iVm § 33 Abs. 3 S. 1 KGSG bestehende sofortige Vollziehbarkeit der Verfügung vom 12. Mai 2017 auszusetzen und so dem Antragsteller zu ermöglichen, wesentliche Teile des Bestands des vom Beigeladenen verwalteten Instituts für Geschichte der Arabisch-Islamischen Wissenschaften an der Johann Wolfgang Goethe-Universität auszuführen, muss erfolglos bleiben, denn bei der allein möglichen summarischen Betrachtung fällt eine Interessenabwägung (Schoch/Schneider/Bier, VwGO, 33. EL Juni 2017, § 80 Rn. 372 ff.) gegen den Antragsteller aus:

[10] Die angegriffene Verfügung erweist sich weder als offensichtlich rechtmäßig noch als offensichtlich rechtswidrig. Da sichergestellte Sachen nicht rechtsgeschäftlich nach den $\S \S 688$ ff. BGB (ggf. in Verbindung mit § 62 S. 2 HVwVfG ) in Verwahrung gegeben werden, sondern hier aufgrund der im Wege der Vollzugshilfe ausgeführten Sicherstellung nach § 33 Abs. 1 Nr. 1 Buchstabe a, § 21 Nr. 2 KGSG aufgrund von § 41 Abs. 1 S. 1 HSOG gesetzlich begründet in Verwahrung zu nehmen sind, das Verwahrungsverhältnis also keinen Rechtsgrund in sich selbst tragen kann, kommt es für die Dauer der Verwahrung auf das Fortbestehen des Sicherstellungsgrundes an, um die fortdauernde Duldungsverpflichtung rechtfertigen zu können ( VG Frankfurt, Urteil vom 9. März 2016 - 5 K 1868/15.F -, juris Rn. 16). Die angegriffene Verfügung wirft Fragen europäischen wie nationalen Rechts auf, die eingehender Feststellungen im Klageverfahren bedürften. Der Regelungsgegenstand der Verfügung betrifft "Kulturgut" im Sinne von § 2 Abs. 1 Nr. 10 KGSG, also eine „bewegliche Sache oder Sachgesamtheit von künstlerischem, geschichtlichem oder archäologischem Wert oder aus anderen Bereichen des kulturellen Erbes, insbesondere von paläontologischem, ethnographischem, numismatischem oder wissenschaftlichem Wert“. Der Begriff des "Kulturguts“ ist weit gefasst, da er sowohl den deutschen Kulturgutbegriff als auch die Kulturgutbegriffe der UNESCO- und EU-Regelwerke umfassen soll (Bundestags-Drucksache 18/7456 S. 59 <zu Nr. 9 des Entwurfs>). Nach dem durch Art. 1 Nr. 6 Buchstabe a Doppelbuchstabe bb des Gesetzes zur Änderung des Grundgesetzes vom 28. August 2006 (BGBI. I S. 2034) eingefügten Art. 73 Abs. 1 Nr. 5a GG hat der Bund die ausschließliche Gesetzgebungskompetenz für "den Schutz deutschen Kulturgutes gegen Abwan- 
derung ins Ausland". Ob "deutsches Kulturgut" nur originär deutsches Kulturgut meint oder aber auch Kulturgut, das sich im Inland mit Zuordnungswillen zum deutschen Herrschaftsbereich befindet - die Motive des verfassungsändernden Gesetzgebers verhalten sich hierzu nicht, vgl. Bundestags-Drucksache 16/813 S. 12, Bundestags-Drucksache 16/2069, insbes. S. 5 zum Ausschuss für Kultur und Medien; Uhle in Maunz/Dürig, GG Art. 73 Rn. 131) - und wie weit somit die Gesetzgebungskompetenz des Bundes reicht, kann hier dahingestellt bleiben, denn wegen der beabsichtigten Ausfuhr aus dem Zollgebiet der Gemeinschaft kommt jedenfalls der Verordnung (EG) Nr. 116/2009 des Rates vom 18. Dezember 2008 über die Ausfuhr von Kulturgütern ( $A B I$. L 39 vom 10.2.2009 S. 1) Anwendungsvorrang zu. Indes beschränkt Art. 2 Abs. 2 UAbs. 3 VO 116/2009 die Möglichkeit, die Ausfuhrgenehmigung zu verweigern, „im Hinblick auf die Ziele dieser Verordnung" darauf, dass "die betreffenden Kulturgüter unter eine Rechtsvorschrift zum Schutz nationalen Kulturguts von künstlerischem, geschichtlichem oder archäologischem Wert in dem betreffenden Mitgliedstaat fallen". Das "nationale Kulturgut" definiert $\S 6$ Abs. 1 KGSG. Hierunter ist Kulturgut zu verstehen, das

- in ein Verzeichnis national wertvollen Kulturgutes eingetragen ist,

- sich in öffentlichem Eigentum und im Bestand einer öffentlich-rechtlichen Kulturgut bewahrenden Einrichtung befindet,

- sich im Eigentum und im Bestand einer Kulturgut bewahrenden Einrichtung befindet, die

- überwiegend durch Zuwendungen der öffentlichen Hand finanziert wird, oder

- Teil einer Kunstsammlung des Bundes oder der Länder ist.

„Kulturgut bewahrende Einrichtung" ist nach § 2 Abs. 1 Nr. 11 KGSG „jede Einrichtung im Bundesgebiet, deren Hauptzweck die Bewahrung und Erhaltung von Kulturgut und die Sicherung des Zugangs der Öffentlichkeit zu diesem Kulturgut ist, insbesondere Museen, Bibliotheken und Archive". Unbeschadet der beantragten Eintragung in ein Verzeichnis national wertvollen Kulturguts dürfte das Institut für Geschichte der Arabisch-Islamischen Wissenschaften an der Johann Wolfgang Goethe-Universität als eine derartige Einrichtung anzusehen sein, so dass mit der Einbringung von Büchern des Antragstellers dessen Eigentum - unabhängig der zwischen den Beteiligten streitigen Frage, ob zivilrechtlich eine Schenkung vollzogen worden sei oder nicht - insofern mit einer öffentlich-rechtlichen Dienstbarkeit belegt worden wäre, als fürderhin ihre Ausfuhr genehmigungspflichtig gestellt wird. Ob es sich dabei, sollte eine Übereignung infolge einer Schenkung verneint werden, um eine hinzunehmende, bloße Inhaltsbestimmung des Eigentums handelt oder darüber hinaus ein Ausgleich vorgesehen sein müsste, bedarf im vorliegenden Verfahren keiner Entschei- dung. Allerdings müssten die betroffenen Kulturgüter im Anhang I der VO 116/2009 benannt worden sein, was hier im Einzelnen insbesondere anhand der Kategorie A10 zu prüfen wäre. Schließlich bedürfte im Hinblick auf die beantragte Eintragung in ein Verzeichnis national wertvollen Kulturguts der Klärung, ob es hier überhaupt um „wesentliche Belange des deutschen Kulturgutbesitzes" im Sinne von § 23 Abs. 2 KGSG geht und diese überwiegen oder die Genehmigung nach § $24 \mathrm{Abs} .5 \mathrm{iVm}$ $\S 21 \mathrm{Nr} .1,3,4$ und 5 KGSG zu erteilen ist.

[11] Die danach vorzunehmende Interessenabwägung fällt zu Lasten des Antragstellers aus, da im Fall einer Ausfuhr vollendete Tatsachen geschaffen würden. Nach Art. 14 Abs. 1 S. 2 GG werden Inhalt und Schranken seines Eigentums „durch die Gesetze" bestimmt, also auch das Kulturgutschutzgesetz und dessen Einschränkungen des Rechtsverkehrs. Würde das Vorliegen einer Ausfuhrgenehmigung fingiert, so dem Antragsteller die Ausfuhr eines wesentlichen Teils des im Institut für Geschichte der Arabisch-Islamischen Wissenschaften an der Johann Wolfgang Goethe-Universität vorhandenen Bestandes in die Türkei ermöglicht und erwiese sich, ungeachtet der oben angeführten Fragestellungen, die Klage als unbegründet, so wäre eine praktisch durchsetzbare Rechtsgrundlage für die Rückschaffung des ausgeführten Kulturguts nicht ersichtlich. Verbliebe die sichergestellte Sachgesamtheit dagegen für die Dauer des Klageverfahrens im Bundesgebiet, so wäre zwar temporär die Verfügungsbefugnis des Antragstellers (schon wegen der Wirkung der Sicherstellung als Veräußerungsverbot im Sinne von § 136 BGB nach § 33 Abs. 3 S. 2 KGSG) eingeschränkt, ein endgültiger Verlust aber nicht zu besorgen. Daher überwiegt das öffentliche Vollzugsinteresse das private Aussetzungsinteresse des Antragstellers, mag der weiteren Verfahrensdauer im Hinblick auf das Alter des Antragstellers auch eine besondere Bedeutung zukommen.

[12] Die Kosten des Verfahrens hat der Antragsteller nach $\S 154$ Abs. 1 VwGO zu tragen, da er unterlegen ist. Die außergerichtlichen Kosten der Beigeladenen sind nach § 162 Abs. 3 VwGO für erstattungsfähig zu erklären, da die Beigeladene einen eigenen Antrag gestellt hat (BI. 164 d.A.) und somit nach $\S 154$ Abs. 3 Halbsatz 1 VwGO ein Kostenrisiko eingegangen ist.

[13] Die Streitwertfestsetzung beruht auf $\S 53$ Abs. 2 Nr. 2, $\S 52$ Abs. 1 GKG. In Ausübung seines Ermessens nimmt das Gericht die Wertgruppe von 50000 Euro des Anhangs I B VO 116/2009 an, ermäßigt den Streitwert aber nach Nr. 1.5 S. 1 des Streitwertkatalogs 2013 auf die Hälfte. 